\title{
Assessing Cobalt Metal Nanoparticles Uptake by Cancer Cells Using Live Raman Spectroscopy
}

This article was published in the following Dove Press journal:

International Journal of Nanomedicine

\author{
Erwan Rauwel (iD) ${ }^{1, *}$ \\ Siham Al-Arag iD ${ }^{2, *}$ \\ Hamideh Salehi (iD ${ }^{2}$ \\ Carlos $O$ Amorim (D) ${ }^{3}$ \\ Frédéric Cuisinier ${ }^{2}$ \\ Mithu Guha ${ }^{4}$ \\ Maria S Rosario 5 \\ Protima Rauwel' \\ 'Institute of Technology, Estonian \\ University of Life Sciences, Tartu, Estonia; \\ ${ }^{2}$ LBN, University of Montpellier, \\ Montpellier, France; ${ }^{3} \mathrm{Dpt}$. Of Physics \& \\ CICECO - Aveiro Institute of Materials, \\ University of Aveiro, Aveiro, Portugal; \\ ${ }^{4}$ Dpt. Of General \& Molecular Pathology, \\ Faculty of Medicine, University of Tartu, \\ Tartu, Estonia; ${ }^{5} \mathrm{CICECO}$ - Aveiro \\ Institute of Materials, University of \\ Aveiro, Aveiro, Portugal \\ *These authors contributed equally to \\ this work
}

\begin{abstract}
Purpose: Nanotechnology applied to cancer treatment is a growing area of research in nanomedicine with magnetic nanoparticle-mediated anti-cancer drug delivery systems offering least possible side effects. To that end, both structural and chemical properties of commercial cobalt metal nanoparticles were studied using label-free confocal Raman spectroscopy.
\end{abstract}

Materials and Methods: Crystal structure and morphology of cobalt nanoparticles were studied by XRD and TEM. Magnetic properties were studied with SQUID and PPMS. Confocal Raman microscopy has high spatial resolution and compositional sensitivity. It, therefore, serves as a label-free tool to trace nanoparticles within cells and investigate the interaction between coating-free cobalt metal nanoparticles and cancer cells. The toxicity of cobalt nanoparticles against human cells was assessed by MTT assay.

Results: Superparamagnetic Co metal nanoparticle uptake by MCF7 and HCT116 cancer cells and DPSC mesenchymal stem cells was investigated by confocal Raman microscopy. The Raman nanoparticle signature also allowed accurate detection of the nanoparticle within the cell without labelling. A rapid uptake of the cobalt nanoparticles followed by rapid apoptosis was observed. Their low cytotoxicity, assessed by means of MTT assay against human embryonic kidney (HEK) cells, makes them promising candidates for the development of targeted therapies. Moreover, under a laser irradiation of $20 \mathrm{~mW}$ with a wavelength of $532 \mathrm{~nm}$, it is possible to bring about local heating leading to combustion of the cobalt metal nanoparticles within cells, whereupon opening new routes for cancer phototherapy.

Conclusion: Label-free confocal Raman spectroscopy enables accurately localizing the Co metal nanoparticles in cellular environments. The interaction between the surfactant-free cobalt metal nanoparticles and cancer cells was investigated. The facile endocytosis in cancer cells shows that these nanoparticles have potential in engendering their apoptosis. This preliminary study demonstrates the feasibility and relevance of cobalt nanomaterials for applications in nanomedicine such as phototherapy, hyperthermia or stem cell delivery.

Keywords: Raman spectroscopy, cobalt nanoparticles, cancer cells, stem cells, cellular uptake, apoptosis, label-free tool

\section{Introduction}

During the last decades, due to the constant demand of more efficient and smaller devices, metal nanoparticles (MNPs) have attracted considerable attention. ${ }^{1,2}$ The main interest in MNPs comes from their unique chemical and electronic properties arising from their high surface to volume ratio. Magnetic nanoparticles are also attracting a lot of interest as size reduction further affects their magnetic properties. Therefore, MNPs with dimensions smaller than the characteristic dimensions of a single magnetic domain are of more particular interest. As a consequence, such
Correspondence: Erwan Rauwel Email erwan.rauwel@emu.ee
International Journal of Nanomedicine 2020:15 705I-7062

7051

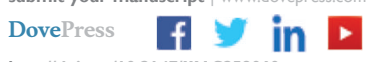

http://doi.org/| $0.2147 / 1$ IN.S258060 
NPs exhibit superparamagnetism (SPM) and exhibit zero coercive field and remanence, ${ }^{3,4}$ thereby making them the best candidates for developing applications in emerging areas like nanomedicine and more particularly in theranostics. ${ }^{5-7}$ The development of nanotechnologies during the last ten years has had a positive impact on several applications in a plethora of fields viz., nanomedicine ${ }^{8,9}$ for magnetic hyperthermia, ${ }^{10,11}$ cancer $^{12,13}$ and orthopedics. ${ }^{14}$ Nanotheranostics, ${ }^{15}$ which combine both therapeutic and diagnostic capabilities at the nanoscale, have broadened the perspectives of personalized medicine. ${ }^{16,17}$ Other targeted applications in the field of nanomedicine have also been investigated, such as photodiagnostic and photothermal anticancer therapy (PTT) using platinum nanoparticles ${ }^{18}$ or gold nanoparticles, ${ }^{19-21}$ as well as nano-loaded stem cell delivery. ${ }^{22}$ Moreover, cancer nanotechnology is a fast growing area of research in nanomedicine mainly because systemic application of anticancer drugs has certain drawbacks with regards to side-effects, non-effective drug concentration at the target area and its en-route degradation. ${ }^{23}$ In that regard, magnetic nanoparticle-mediated anti-cancer drug delivery systems offer added advantages, such as minimized side effects, along with a better control for drug delivery. Therefore, researchers are actively exploring these properties $^{24,25}$ by investigating the interaction between the cell membrane and nanoparticles, which has been the focus of multiple studies. ${ }^{26}$ In fact, different cellular uptake modes can occur and require further understanding in order to develop a suitable treatment. Additionally, the toxicity of metal nanoparticles involved in such uptakes also requires assessment before integrating these nanomaterials into cancer treatment applications.

Raman spectroscopy is a non-invasive method of analysis based on biochemical mapping of the analysed sample area. This technique provides specific information on the unique vibrations of molecules after excitation with a monochromatic laser. Confocal Raman microscopy with its high spatial resolution and unique compositional sensitivity is used as a unique label-free tool to trace drugs and nanoparticles within cells. ${ }^{27-31}$ The cells and their components are analysed via their vibrational spectra owing to differences in their biochemical compositions. The Raman nanoparticle signature therefore enables precise nanoparticle detection in the cell without any labeling. ${ }^{27-29,32} \mathrm{By}$ applying this method, the uptake of Co MNPs by cancer cells and their localization within were investigated.
The Co MNPs are renowned catalysts, more particularly in Fischer-Tropsch reactions. ${ }^{33}$ Reports on Co MNPs investigated for biomedical applications (or in biomedicine) are very scarce and most of the investigations have mainly been carried out with cobalt metal oxide nanoparticles ${ }^{34,35}$ due to the intrinsic pyrophoric properties of Co MNPs. In general, Co MNPs have to be coated with an oxide layer, a carbon or an organic layer in order to suppress their violent and spontaneous oxidation on exposure to air. ${ }^{36}$ Furthermore, Co MNPs with sizes under $8 \mathrm{~nm}$ exhibit superparamagnetic properties, ${ }^{37,38}$ along with a high magnetic saturation, thus making them promising nanomaterials towards applications in emerging areas like nanomedicine and water purification. Therefore, ultrastable non-pyrophoric Co MNPs are necessary for the development of practicable applications. The surfactantfree Co MNPs used for this study did not exhibit any pyrophoric behaviour, which made studying their cellpenetrating abilities against cancer cells achievable.

In this study, we have investigated the interaction between cobalt metal nanoparticles and cancer cells using Raman spectroscopy as a label-free tool to investigate the development of alternate cancer treatment routes.

\section{Materials and Methods Cobalt Metal Nanoparticles}

Cobalt metal nanoparticles are of industrial grade and have been provided by PRO-1 NANOSolutions in the form of a black powder. Before utilization, the Co nanoparticles were dispersed in PBS buffer solution through energetic shaking and then sonicated for 5 minutes in an ultrasonic bath.

\section{Cell Lines}

The following cell lines were used: MCF-7 (ATCC HTB22), HCT 116 (ATCC CCL-247), HEK 293 (ATCC CRL1573), and PPC-1 (ATCC HTB-190). Furthermore, primary mesenchymal stem cells derived from the dental pulp (DPSC) were tested. ${ }^{27-29,32}$ DPSCs were obtained from human wisdom teeth, extracted for orthodontic reasons and recovered from healthy patients (age range: 15-18 years). Written informed consent was obtained from the patients' parents as approved by the local ethical committee (Comité de Protection des Personnes, Montpellier hospital, France). DPSCs were isolated and characterized as previously described. ${ }^{29}$ Cancer cell lines and primary mesenchymal stem cells were grown in DMEM and 
aMEM media, respectively (Dulbecco's and Alpha Modified Eagle's Medium) containing 10\% FBS and 1\% antibiotics (100 $\mu \mathrm{g} . \mathrm{mL}^{-1}$ penicillin Streptomycin) at $37^{\circ} \mathrm{C}$ and $5 \% \mathrm{CO}_{2}$.

\section{PBS Buffer Solution}

PBS buffer solution is composed of $1 \mathrm{~L}$ of distilled water in which $8 \mathrm{~g}$ of $\mathrm{NaCl}, 0.2 \mathrm{~g}$ of $\mathrm{KCl}, 1.44 \mathrm{~g}$ of $\mathrm{Na}_{2} \mathrm{HPO}_{4}$ and $0.24 \mathrm{~g}$ of $\mathrm{KH}_{2} \mathrm{PO}_{4}$ were added. The $\mathrm{pH}$ of the solution was adjusted to 7.4 by addition of $\mathrm{HCl}$.

\section{Structural Study}

X-ray diffraction (XRD) patterns were collected using a Panalytical Empyrean diffractometer with a $\mathrm{Cu} \mathrm{K \alpha l}$ radiation source $(\lambda=0.15406 \mathrm{~nm})$. CHN measurement was carried out on Leco Truspec Micro CHNS Analyzer model $630-200-200$, at temperature of $1075^{\circ} \mathrm{C}$. Carbon, hydrogen and sulphur were measured by infrared absorption and nitrogen was measured by thermal conductivity.

\section{Morphological Study}

High-resolution transmission electron microscopy (HRTEM) was carried out on 80-300 FEI Titan, operating at $300 \mathrm{kV}$, disposing a point to point resolution of $1.4 \AA$.

\section{Magnetic Measurements}

Magnetic measurements were performed using a Quantum Design MPMS3 SQUID magnetometer, using its VSM measurement option, at $300 \mathrm{~K}$, using magnetic fields ranging from -70 and $+70 \mathrm{kOe}$. The remnant magnetic fields of the superconducting magnet used as the magnetic field source (about $22 \mathrm{Oe}$ after reaching $70 \mathrm{kOe}$ ) were considered during the estimation of the coercive field.

\section{Raman Spectroscopy}

Raman spectra were collected using a WITec Confocal Raman Microscope System alpha 300R (WITec Inc., Ulm, Germany). Excitation in confocal Raman microscopy is generated by a frequency-doubled Nd:YAG laser (New-port, Irvine, CA, USA) at a wavelength of $532 \mathrm{~nm}$, with $50 \mathrm{~mW}$ maximum laser output power in a single longitudinal mode. The incident laser beam is focused onto the sample through a $60 \times$ NIKON water immersion objective having a numerical aperture of 1.0 with a working distance of $2.8 \mathrm{~mm}$ (Nikon, Tokyo, Japan). The laser power after the objective is $15 \mathrm{~mW}$ but the power absorbed by cells in PBS is lower.

The spatial and depth resolutions are $300 \mathrm{~nm}$ and $1 \mu \mathrm{m}$, respectively. The mixed scattered radiation passes through an edge filter to block the Rayleigh radiation from the Raman signal, which is then directed to the electron multiplying charge coupled device camera EMCCD (DU 970N-BV353, Andor, Hartford, USA). The electron multiplying charge coupled device chip size was $1600 \times 200$ pixels, the camera controller was a 16-bit A/D converter operating at $2.5 \mathrm{MHz}$. The acquisition time of a single spectrum was set to $0.5 \mathrm{sec}$. An area of $150 \times 150$ points per image was recorded leading to the acquisition of 22500 spectra for one image. The advantage of our analysis method using EMCCD (Electron multiplying CCD) is the high speed of scan. Each pixel can be recorded with a very good signal-to-noise ratio at low integration times. For the high spatial resolution of the system $(300 \mathrm{~nm})$, a complete image of a whole cell is recorded for a better analysis. Data acquisition and processing were performed using Image Plus 5.2 software (Ulm, Germany) from WITec.

The analysis is based on two methods. The first method provides integrated Raman intensities in specific regions in particular $\mathrm{C}-\mathrm{H}$ stretching modes. The integrated Raman intensities of $\mathrm{C}-\mathrm{H}$ stretching mode $\left(2800-3000 \mathrm{~cm}^{-1}\right)$ represent the lipid-protein distribution in the cells. Data processing is performed using Image Plus software from WITec. Each image regarding these integrated intensities could provide a map of the region. Using a color-coded reference table, bright yellow hues indicate the highest intensities while orange hues the lowest integrated intensities of the chosen region. The second analysis method is the K-mean cluster analysis (KMCA), which separates data into k-mutually exclusive clusters and can run multiple times. KMCA was realized using the WITec Project Plus software.

For Raman experiments, 2x105 MCF7 cells were cultivated for $24 \mathrm{~h}$ onto polished and disinfected calcium fluoride $\mathrm{CaF}_{2}$ (Crystran Ltd, Dorset, UK) substrates in $35 \mathrm{~mm}$ petri dishes. Nanoparticles were suspended in pure distilled deionized water. The dissolved nanoparticle solution was then added to the cell culture medium at $2 \mu \mathrm{g} / \mathrm{mL}$ concentration for 1-hour incubation. The $\mathrm{CaF}_{2}$ substrates with adhered cells are fixed with 2\% PFA (paraformaldehyde) after thorough rinsing with 1x PBS. Cells were kept in 5mL PBS and transferred directly for Raman measurements.

\section{Toxicity Study}

Cytotoxicity of different concentrations of cobalt nanoparticles was assessed via MTT assay. Human embryonic kidney (HEK) or PPC-1 cells were seeded on day 0 at 
a density of 1000 per well in 96 well microtiter plates. On day 1, cobalt nanoparticles at different concentrations were added. After a $24 \mathrm{~h}$ incubation period, the medium containing nanoparticles were removed from the plate to ensure that no nanoparticles remain in the solution and avoid overlapping or hinder MTT assay. After $24 \mathrm{~h}$, 3-(4,5-dimethylthiazol-2-yl)-2,5-diphenyltetrazolium bromide (MTT) was added to each well $(0.5 \mathrm{mg} / \mathrm{mL}$; SigmaAldrich) and plates were maintained at $37^{\circ} \mathrm{C}$ for 2 hours. The medium was then discarded; DMSO was added to each well to lyse the cells. Absorbance was measured at $530 \mathrm{~nm}$ using a multiwall spectrophotometer (Tecan, microplate reader). All MTT assays were repeated twice.

\section{Results and Discussion}

\section{Morphological Characterizations}

In Figure 1A, the X-ray diffraction pattern obtained from the Co nanopowder shows that cobalt nanoparticles are highly crystalline with a face-centered cubic (Fm3m) crystal structure $(\mathrm{a}=3.5447 \AA)$ and a crystallite average diameter of $2.5 \mathrm{~nm}$ (JCPDS 15-806). ${ }^{39}$ The $<111>,<200>$ and $<220>$ diffraction peaks are in good agreement with the crystalline cubic Co metal (PDF No. 79-1770). ${ }^{39}$

The TEM micrographs of Figure $1 \mathrm{~B}$ and $\mathrm{C}$ provide an overview of the cobalt metal nanoparticles investigated in this study. In Figure 1B, some of the Co MNPs are spherical and monodispersed on the carbon-coated grid used
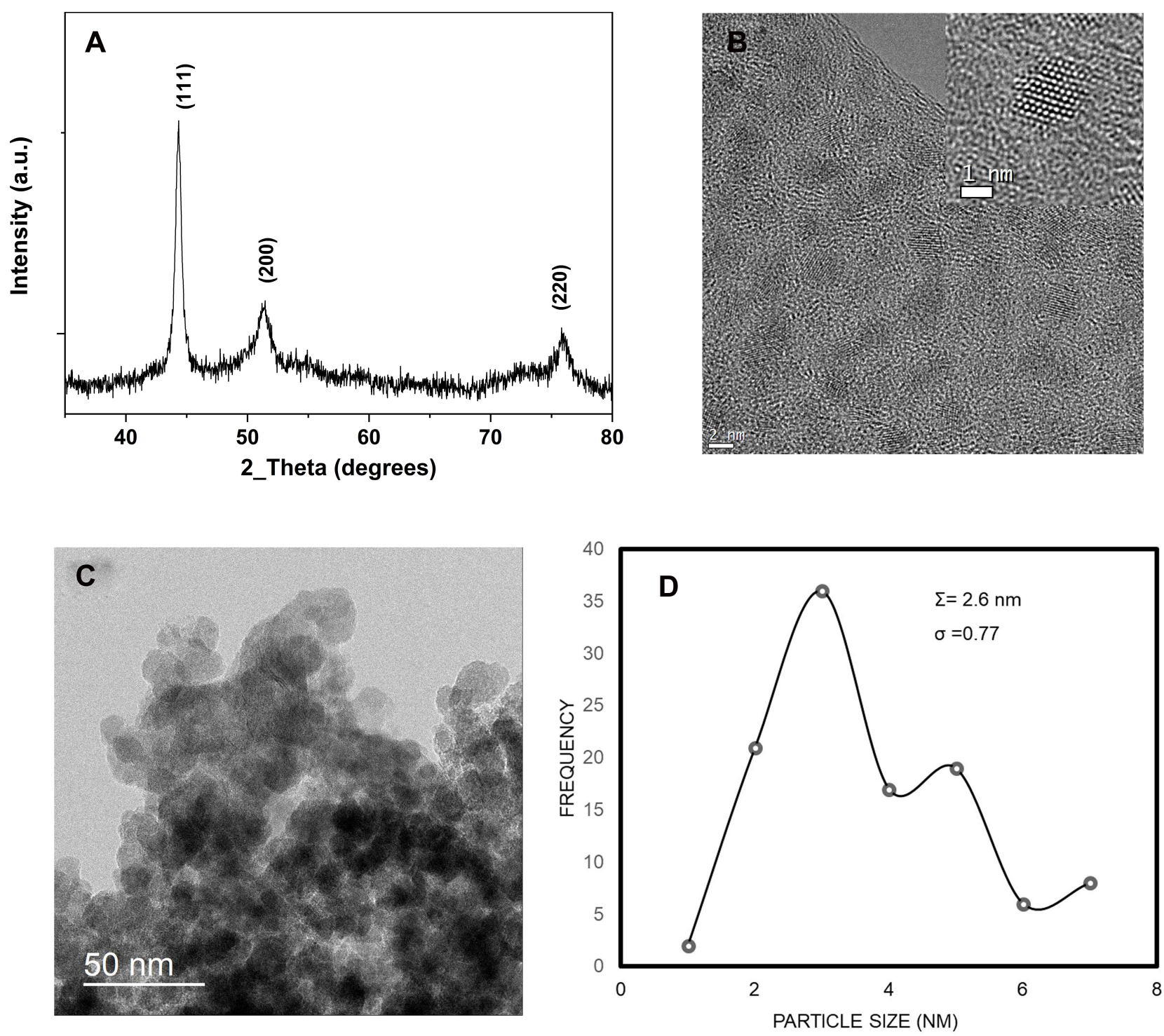

Figure I (A) XRD pattern of Co metal nanoparticles used in the study, (B) high-resolution overview of cubic Co metal nanoparticles of $5 \mathrm{~nm}$ diameter, inset is a highresolution TEM image of one cobalt nanoparticle (C) low magnification TEM overview of cubic Co metal nanoparticles agglomerated, (D) size distribution histogram. 
for TEM observation. They are all crystalline as observed from the lattice fringes on the same image. In the inset, a high-resolution micrograph of one highly crystalline nanoparticle is provided. The nanoparticle is oriented in the $<110>$ direction as indicated by the point-to-point resolution of the atomic columns. Contrary to Figure 1B, some of the Co nanoparticles in Figure 1C tend to agglomerate into larger clusters mainly because they are surfactant free. The size distribution histogram in Figure 1D provides a mean nanoparticles size of $2.6 \mathrm{~nm}$ with a low standard deviation of 0.77 .

The cleanliness of the Co MNPs surfaces and the detection of surfactants or organic species were investigated using carbon-hydrogen-nitrogen $(\mathrm{CHN})$ measurements. After analysing $2.218 \mathrm{mg}$ of Co MNPs, 0.451 weight $\%$ of carbon and 0.239 weight $\%$ of nitrogen was obtained; neither hydrogen nor sulphur was detected. This analysis suggests that carbon and nitrogen detected are mainly due to exposure to air. In addition, since there is no organic coating on the surface of the Co MNPs, therefore, the effect of direct interaction of Co MNPs with cancer cells can be straightforwardly evaluated.

\section{Magnetic Properties}

The magnetic properties of the Co nanoparticles were investigated and the magnetic field dependence magnetization (M-H) hysteresis loop at 300K was measured. A typical $\mathrm{M}-\mathrm{H}$ loop measured at $300 \mathrm{~K}$, and normalized to the Co mass content, is presented in Figure 2. The M-H curve at $300 \mathrm{~K}$ displays a saturation magnetization at around $5 \mathrm{kOe}$

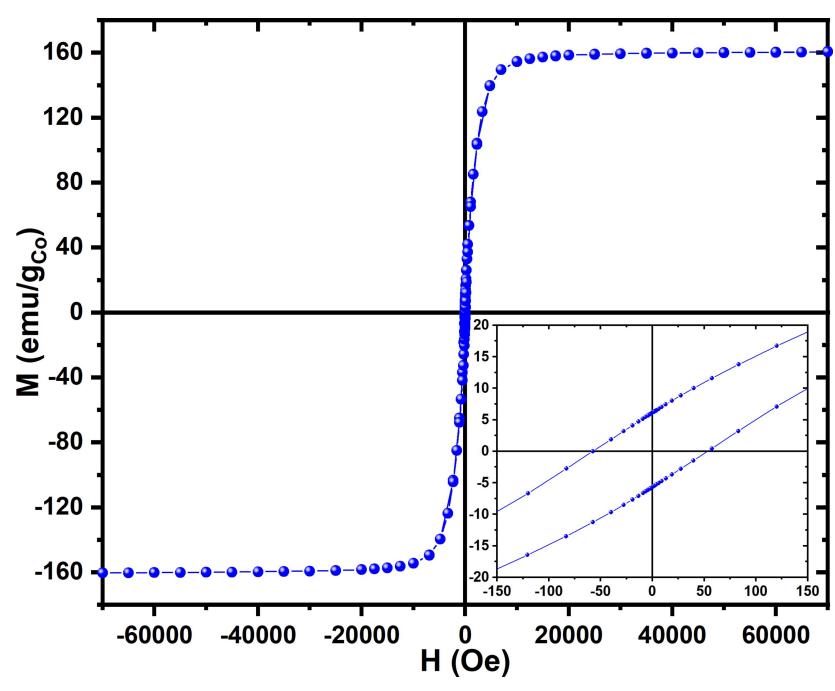

Figure $2 \mathrm{M}-\mathrm{H}$ hysteresis loop measured at $300 \mathrm{~K}$ for Co nanoparticles, inset shows the details of the measurement around the origin. with an estimated coercivity $\mathrm{H}_{\mathrm{C}}<76 \mathrm{Oe}$ (inset of Figure 2). The saturation magnetization (MS) at $300 \mathrm{~K}$ is $160.4 \mathrm{e} . \mathrm{m} . \mathrm{u} . / \mathrm{g}_{\mathrm{Co}}$, which is comparable to the theoretical value of the saturation magnetization of $163 \mathrm{e} . \mathrm{m} . \mathrm{u} . / \mathrm{g}_{\mathrm{Co}}$ for Co metal. ${ }^{37,40,41}$ This further confirms that the major content $(>90 \%)$ of our samples is metallic cobalt. The investigations of the magnetic properties of the Co nanomaterials reveal that the Co MNPs exhibit a mixture of superparamagnetic and a soft ferromagnetic behaviour.

\section{Raman Spectra Study}

In order to study the interaction between Co MNPs and MCF7 breast cancer cells, the Raman scattered light from freestanding Co MNPs was analysed. Figure $3 \mathrm{~A}$ and B are the optical images of the Co MNPs analysed by Raman spectroscopy. The two Raman patterns (calculated as average spectra) obtained at low and high laser power $5 \mathrm{~mW}$ and $20 \mathrm{~mW}$, respectively (Figure 3C) were also compared. The Co MNPs spectrum is characterized by three main peaks centred at 474 , 512,608 and $680 \mathrm{~cm}^{-1}$. These peaks correspond to the $\mathrm{Eg}$, $\mathrm{F}_{2 \mathrm{~g}}{ }^{1}, \mathrm{~F}^{2}{ }_{2 \mathrm{~g}}$, and $\mathrm{A} 1$ modes of single-crystal $\mathrm{Co}_{3} \mathrm{O}_{4}{ }^{42,43}$

Although the laser power is less than $20 \mathrm{~mW}$, it is nevertheless sufficient for spontaneous combustion of the Co nanoparticles to occur (Figure 3B). This could be attributed to the absence of organic coating on their surfaces. Under low laser power, the Raman pattern corresponds to face-centred cubic cobalt metal as illustrated by the blue spectrum in figure $3 c .{ }^{44,45}$ The Raman pattern in Figure $3 \mathrm{C}$ (red spectrum) corresponds to the $\mathrm{Co}_{3} \mathrm{O}_{4}$ crystalline structure. Consequently, it confirms that the laser irradiation provokes the spontaneous oxidation of the Co MNPs. Therefore, the laser power was reduced to less than $5 \mathrm{~mW}$ during the study. The Raman spectrum at low energy served as a reference for the identification of free-standing Co MNPs in the PBS solution. Co MNP were stabilized in the DMEM medium containing MCF7 breast cancer cells for 1 hour, which was sufficient to bring about the diffusion of nanoparticles within the MCF7 breast cancer cells.

Raman spectroscopy enables identifying two different signals from the Co MNPs. In Figure 4, the blue spectrum pertains to the extracellular nanoparticle signature, as the $\mathrm{C}-\mathrm{H}$ group belonging to cellular proteins is absent, while in the red spectrum the $\mathrm{C}-\mathrm{H}$ peak at $2800 \mathrm{~cm}^{-1}$ and $\mathrm{OH}$ at $3200 \mathrm{~cm}^{-1}$ in the presence of nanoparticles refers to intracellular nanoparticles. Detecting the spectra from the voxels combined with advanced data analysis therefore enables us to distinguish between extracellular and intracellular nanoparticles (Figure 4). 


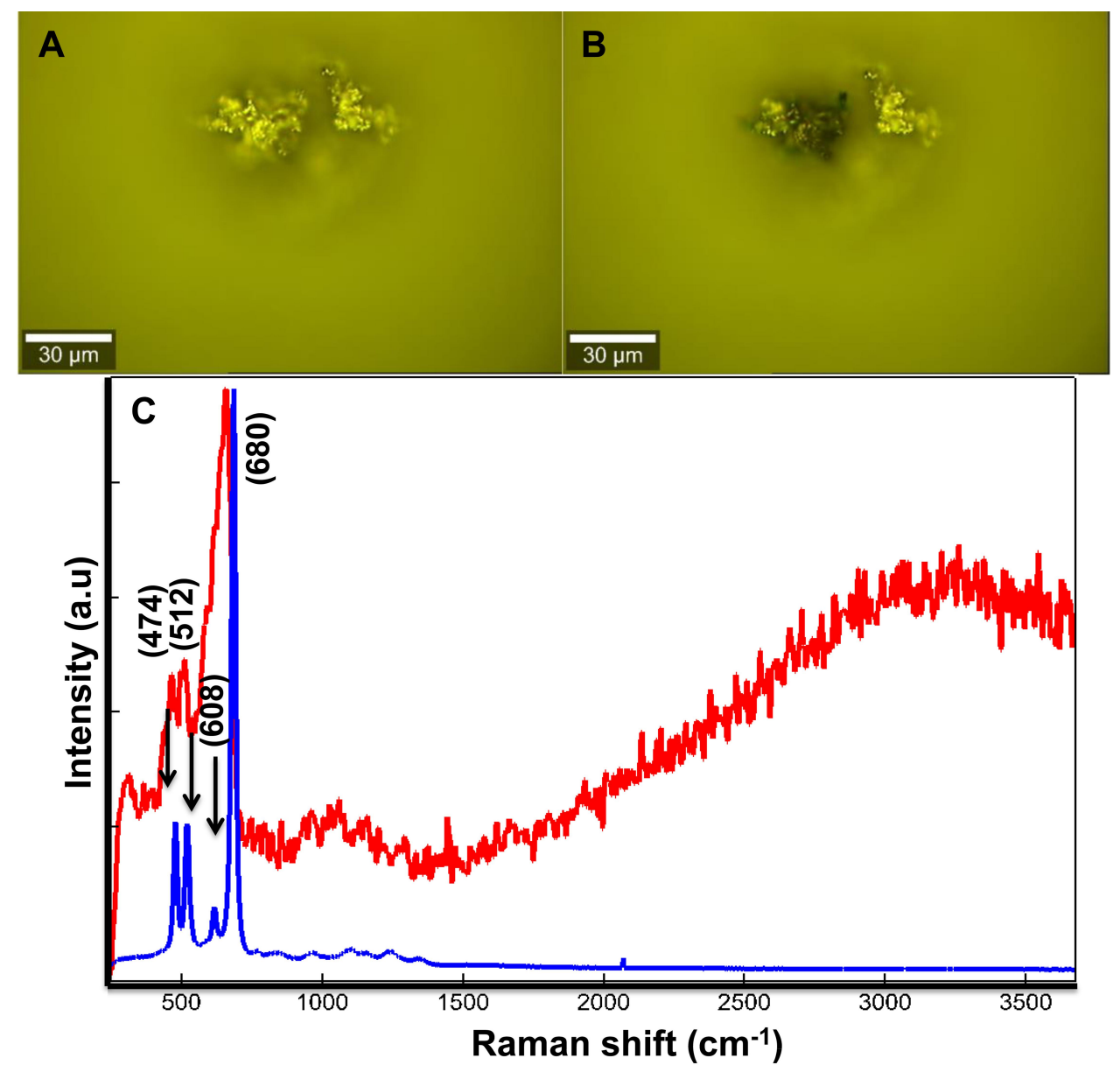

Figure 3 Optical images of agglomerated Co MNPs studied by Raman spectroscopy with a laser power of (A) $5 \mathrm{~mW}$ and (B) $20 \mathrm{~mW}$, (C) comparison of the Raman pattern obtained from Co MNPs powder with $532 \mathrm{~nm}$ laser excitation under low power $5 \mathrm{~mW}$ indicated by blue spectrum (A) to higher laser power $20 \mathrm{~mW}$ indicated by red spectrum (B).

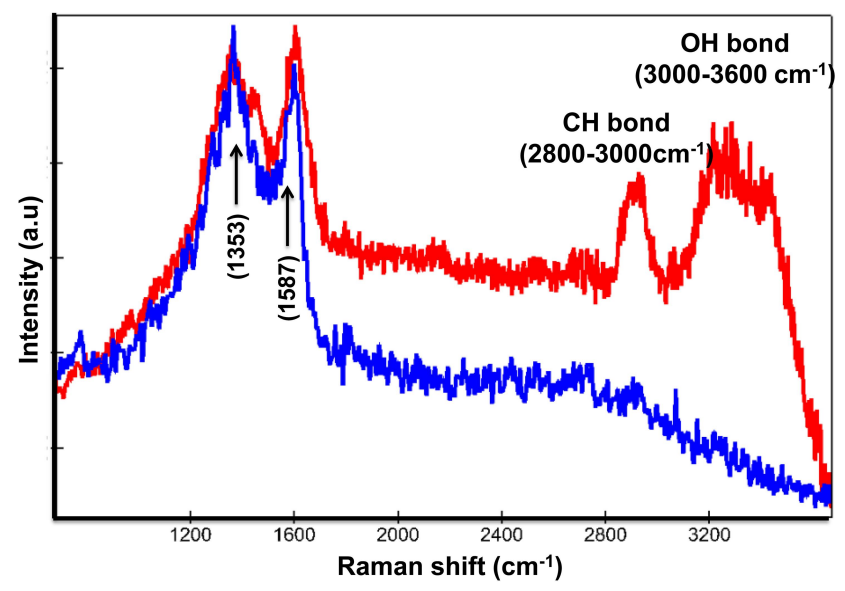

Figure 4 Raman patterns obtained from Co MNPs outside the MCF7cells (blue spectrum) and inside MCF7cells (red spectrum) with $532 \mathrm{~nm}$ laser excitation under $5 \mathrm{~mW}$.

Figure $5 \mathrm{~A}$ and $\mathrm{B}$ exhibit two reconstructed images of MCF7 breast cancer cells incubated with Co MNPs. Figure $5 \mathrm{~A}$ is the integrated Raman image of intensities in the $2800-3000 \mathrm{~cm}^{-1}$ region, which is the lipid-protein region of cells. In Figure 5B, using k-mean cluster analysis (KCMA), we were able to distinguish between the particles (green pixels indicated by arrows). Figure $5 \mathrm{C}$ presents the Raman spectrum of Co MNPs, with the lipid protein peak (2800-3200 $\mathrm{cm}^{-1}$ ), inside MCF7 breast cancer cells, while Figure 5D shows the Raman spectrum of Co MNPs outside the cells.

Figure 6 displays the 1 hour incubation of Co MNPs with different cell types: breast cancer cells MCF7 (Figure 6A and B), colorectal cancer cells HCT116 (Figure 6C and D) and dental pulp mesenchymal stem cells DPSC (Figure 6E to $\mathrm{H}$ ). All the cells exhibit membranous cellular localization of the nanoparticles, either on the plasma membrane or on the nuclear membrane, within a span of 1 hour of nanoparticle exposure. Co MNPs seem to favour membrane anchorage when put in contact with cells, rather than cytoplasm anchorage. Noticeably in the case of HCT116 cells (Figure 6C), 


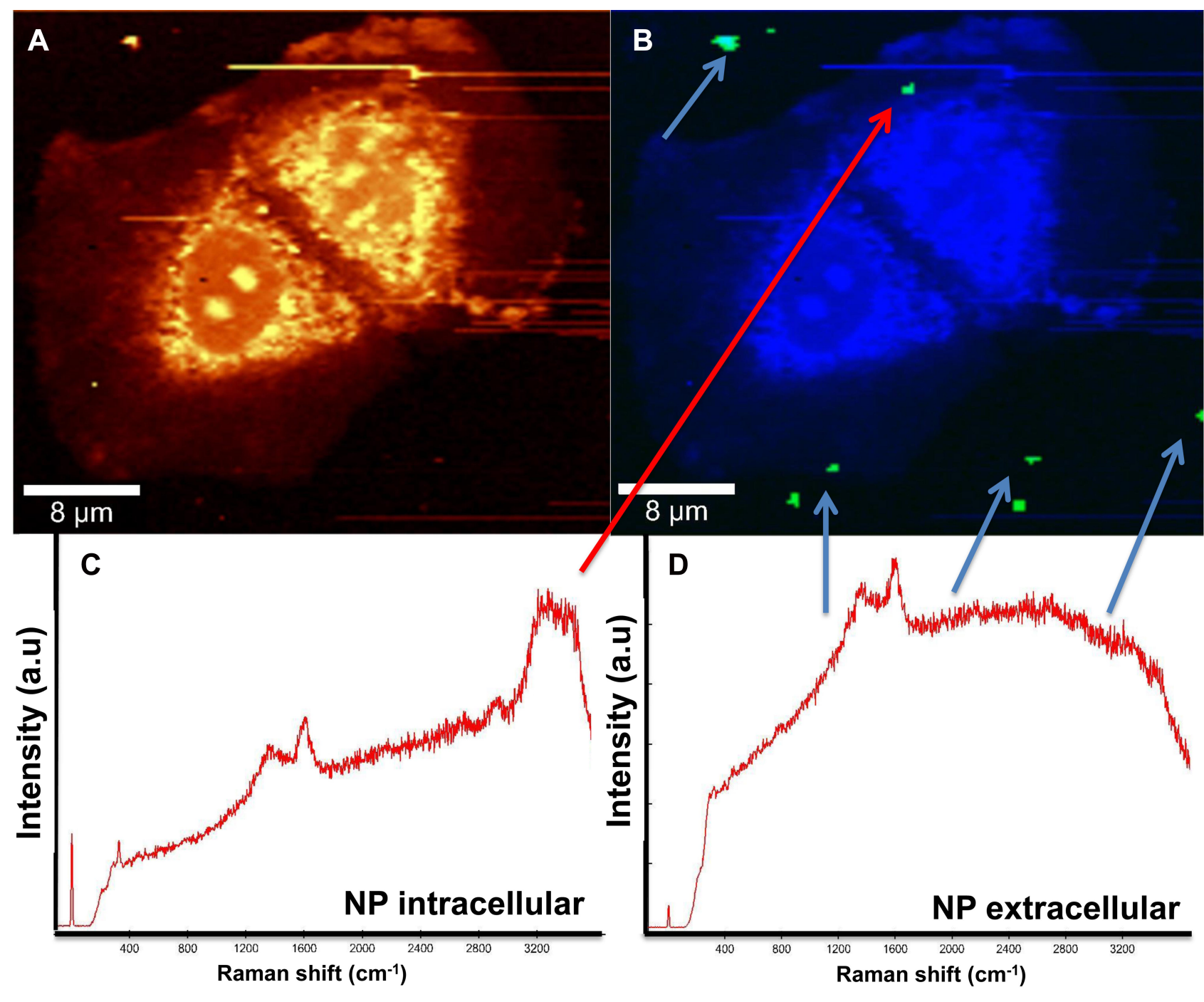

Figure 5 MCF-7 cells incubated with Co nanoparticles, (A) integrated Raman intensities in the $2800-3000 \mathrm{~cm}^{-1}$ region of cells, (B) Raman reconstruction of image A using KMCA to detect Co nanoparticles (green pixels indicated by arrows), (C) Raman spectrum of Co MNPs inside MCF7 cells (indicated by the red arrow), and (D) spectrum outside of the cells (blue arrows).

intracellular nanoparticles are fixed to the outer cell membrane surfaces, while subsequent internalization of nanoparticles into the cells is expected with higher incubation time (after 1 hour). This implies that a longer incubation time would be necessary for the nanoparticles to penetrate the cell nucleus.

\section{Cytotoxicity Study}

It is also important to investigate the cytotoxicity of these cobalt nanoparticles against human cells before applying them in nanomedicine. The Co element is known to be well tolerated by the human body that can easily eliminate cobalt as it is a non-accumulating metal. ${ }^{46}$ Therefore, the cytotoxicity of PBS solutions containing Co MNPs was investigated on Human embryonic kidney HEK 293 cells. $^{47}$ Different concentrations of Co MNPs dispersed in PBS solution were prepared and studied by MTT assay. In Figure 7, MTT assays were performed on Co MNP solutions of concentration ranging from $0.5 \mu \mathrm{g}$ to $120 \mathrm{mg} / \mathrm{L}$ for $24 \mathrm{~h}$ and the data were analysed using one way ANOVA followed by Dunnett's post hoc test. Differences were considered significant if $\mathrm{p}<0.05$. Figure 7 shows the mean \pm standard error of mean (SEM) of duplicate measurements of a representative sample of three independent experiments. This toxicity study towards HEK 293 cells shows that mortality rate is very low for the studied concentrations of cobalt nanoparticles $(120 \mathrm{mg} / \mathrm{L}(0.12 \mathrm{ppm}))$ and is around $17 \%$. For low concentrations, no visibly high toxicity was detected against HEK 293 cells. A Similar cytotoxicity study was performed on primary prostatic cancer cell line (PPC-1). Figure 7B presents MTT assays performed on Co 

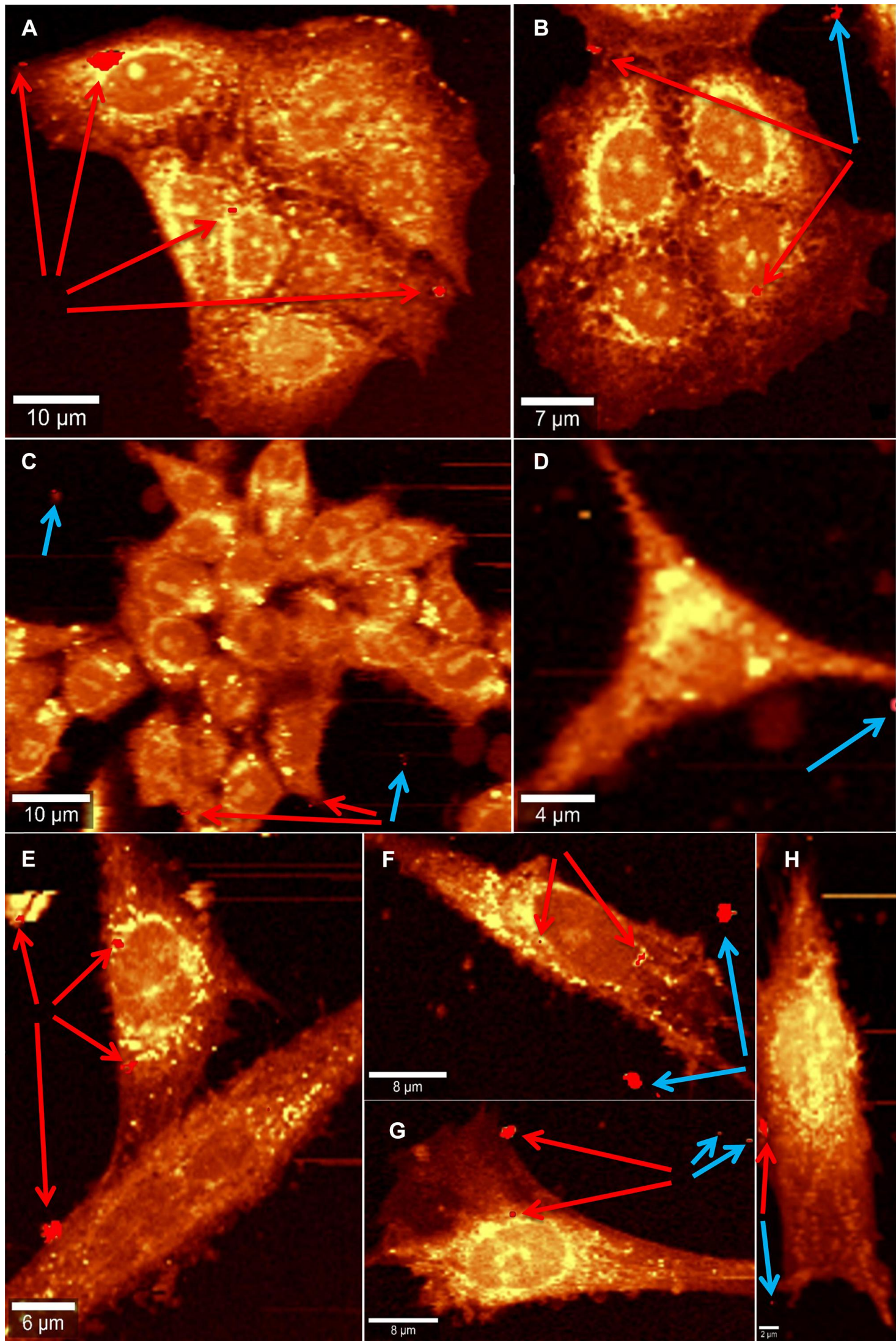

Figure 6 Raman reconstructed images showing Co MNPs inside cells (indicated by red arrows) and outside cells (indicated by blue arrows) when incubated for I hour with breast cancer cells MCF7 (A and B), colorectal cancer cells HCTII6 (C and D) and with dental pulp mesenchymal stem cells DPSC (E-H) 
MNPs solutions of concentrations ranging from $0.5 \mu \mathrm{g} / \mathrm{L}$ to $50 \mathrm{mg} / \mathrm{L}$ for $24 \mathrm{~h}$.

The toxicity study on PPC-1 cells indicates that the mortality rate is over $64 \%$ for a concentration of cobalt nanoparticles of $0.5 \mu \mathrm{g} / \mathrm{L}$ and shows a higher mortality rate (over $79 \%$ ) for a concentration of $50 \mathrm{mg} / \mathrm{L}$. These results therefore demonstrate that Co MNPs are potential candidates for the treatment of cancer.

\section{Discussion}

In this study, we demonstrate that Raman spectroscopy can be used as a label-free tool in order to investigate the interaction between Co MNPs and different cell types. The goal was to examine whether the Co MNPs were able to penetrate the cell membranes of cancer cells. Raman microscopy enables the efficient label-free tracing of the Co MNPs inside the cells by differentiating them from the free-standing Co MNPs outside the cells. Our study also allowed localizing the nanoparticles inside the cell by Confocal Raman data analysis.

In this study, we validate that Co MNPs easily penetrate the cell membrane of cancer cells leading to their potential apoptosis. Endocytosis is a normal process in living cells and is also known as cellular uptake. Cellular uptake of NPs involves distinctive mechanisms. In addition, cancer cells exhibit different uptake velocities and behaviours than healthy human cells. ${ }^{48,49}$ Endocytosis is normally used by cells for uptake of proteins and other macromolecules but also applies to NPs. It has been observed that NP uptake is usually more pronounced in cancer cells $^{50}$ and the internalization rate is also higher for more aggressive cancer cells than healthy cells, ${ }^{51}$ which can be considered as an asset for treatment options based on nanoparticle uptake. Raman spectroscopy then emerges as a convenient and rapid method towards investigating the interaction between nanomaterials and cells. This study also highlights that it is possible to induce the fast oxidation of the Co MNPs under laser irradiation of $532 \mathrm{~nm}$ wavelength with the power of $20 \mathrm{~mW}$. Using phototherapy or bringing about the destruction of the cancer cells through the combustion of Co MNPs introduced within a tumour is therefore valid in such a scenario. The magnetic properties exhibited by the Co MNPs also offer the possibility to target a specific location in the body through magnetic field assistance and combine phototherapy with hyperthermia therapy. This is presently under investigation and may offer new routes towards the development of cancer theranostics in combination with hyperthermia and phototherapy. The toxicity of the Co MNPs used in this study was also investigated in order to evaluate the validity of a potential application for cancer treatment. Furthermore, the low cytotoxicity exhibited towards HEK 293 cells indicates that the mortality rate is very low even for high Co nanoparticle concentration of $120 \mathrm{mg} / \mathrm{L}$. Conversely, the toxicity against PPC-1 cancer is very high, even at concentrations as low as $5 \mu \mathrm{g} / \mathrm{L}$. This establishes the high potential of cobalt metal nanoparticles for applications in nanomedicine, more particularly for cancer therapy, considering the rapid uptake of Co MNPs by cancer cells. Selectivity combined with hyperthermia capabilities of the Co MNPs make them promising candidates for the development of targeted therapies such as drug delivery. Similar results have been recently reported with Au MNPs against breast cancer cells, in which positively charged Au MNPs caused abrupt destruction of MDA-MB -231 cells. $^{52}$ In addition, the study reports that treatment with Au NPs makes MDA-MB-231 cells sensitive to 5-fluorouracil due to epigenetic changes. In effect, DNA fragmentation was detected with Au MNPs; however, in this present study, Raman spectroscopy did not show DNA fragmentation with Co MNPs. Therefore, the Co MNPs toxicity mechanism needs to be further investigated.

In addition, we have studied the uptake of nanoparticles by mesenchymal stem cells (MSCs). New research has revealed encouraging results for the application of MSCs as cellular carriers of therapy towards cancer tissue. "Nano-engineered" MSCs have been applied in different disciplines: in delivering therapeutics to tissues, in homing to cancer or inflammatory sites and in cancer imaging and immunotherapy. This "non-genetic" engineering approach, provided by nanoparticle loading, has unique advantages in cancer therapy and is complementary to existing genetic cell manipulation. ${ }^{22,53}$ MSCs carrying nanoparticles (MSCs-NPs) can be used to achieve targeted anti-cancer effects. Current results indicate that MSCs-NPs combine tumour tropism and penetrative abilities of MSCs, in addition to controlled NP release. ${ }^{54}$

Dental pulp constitutes an easily accessible and noninvasive source of mesenchymal stem cells when compared to other adult stem cell sources. Dental pulp stem cells (DPSCs) have been previously investigated for their resistance to anti-cancer drug and have revealed potential for applications in drug delivery and reduce chemotherapy side effects. ${ }^{29,32}$ For these reasons, we investigated the DPSCs potential uptake ability for nanoparticle delivery (Figure 6E-H). MSCs manifest high resistance to the 

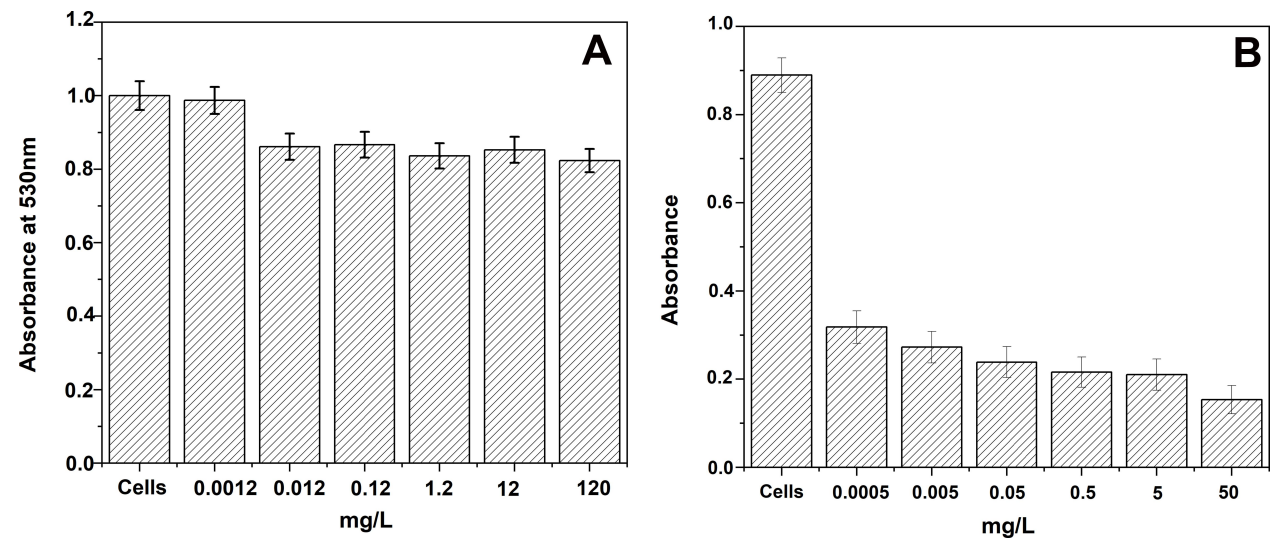

Figure 7 Toxicity test of cobalt metal nanoparticles (A) on HEK 293 cells, (B) on PPC-I cells.

cytotoxic effect of internalized nanoparticles, as was shown with HEK 293 cell viability tests, up to high concentrations of Co MNPs (Figure 7A). On one hand, nanoparticles fixed to cell membranes have ignorable cytotoxicity to MSCs (favourable localization). On the other hand, as in the case of anticancer drug loading in cells, limited intracellular spacing makes it difficult to administer high therapeutic doses. Nanosized particles in contrast may help to maximize therapeutic loading and allow MSC migration followed by nanoparticle release into target tissues.

\section{Conclusion}

We have studied the interaction between surfactant-free cobalt metal nanoparticles with cancer cells. The investigation demonstrated the ability of non-invasive, label-free confocal Raman spectroscopy to discriminate freestanding cobalt metal nanoparticles present in a PBS solution from the nanoparticles inside the cancer cells. Raman spectroscopy enables accurate localization of the Co metal nanoparticles in a cellular environment, owing to their chemical fingerprint and advanced data analysis methods.

The facile endocytosis in cancer cells also shows that these cobalt metal nanoparticles have a potential for inducing apoptosis of cancer cells. The fact that cobalt is a non-accumulative element for the human body, contrary to iron and copper, makes Co MNPs a possible candidate for cancer treatment. Furthermore, the toxicity test performed on HEK 293 cells showed a very low toxicity of the Co MNP for concentrations lower or equal to $120 \mathrm{mg} / \mathrm{L}$. Moreover, based on the marked tropism of MSCs toward tumours, it is expected that MSCs loaded with Co MNPs can target tumours and tumour metastases after systemic injection. The loaded Co MNPs could be released into the tumour niche through different routes: phototherapy, hyperthermia, or stem cell delivery.

The study also highlights that under a laser irradiation of $20 \mathrm{~mW}$ with a wavelength of $532 \mathrm{~nm}$ it is possible to locally induce combustion of the Co MNPs inside the cells, which opens new routes for cancer phototherapy. This preliminary study demonstrates the considerable capability of cobalt nanomaterials for applications in nanomedicine and further investigations are ongoing. All in all, Raman spectroscopy offers a new tool towards such implementations.

\section{Acknowledgments}

PRO-1 NANOSolutions is gratefully acknowledged for providing the cobalt metal nanoparticles used in this study. The authors thank Dr. Céline Gongora (Cancer Research Institute IRCM Montpellier, France) for providing the colorectal cancer cell line. The Center for Electron Nanoscopy at the Technical University of Denmark is acknowledged for access to their transmission electron microscopes. This research was funded by the European Regional Development Fund project EQUiTANT, grant number TK134 (F180175TIBT). This work was developed in the scope of the project CICECO-Aveiro Institute of Materials (ref. no. FCT UID/CTM/50011/2013), financed by national funds through FCT/MEC and co-financed by FEDER under the PT2020 Partnership Agreement.

\section{Disclosure}

The authors report no conflicts of interest for this work. 


\section{References}

1. Berry CC, Curtis ASG. Functionalisation of magnetic nanoparticles for applications in biomedicine. J Phys D: Appl Phys. 2003;36(13): R198-R206. doi:10.1088/0022-3727/36/13/203

2. Jeevanandam J, Barhoum A, Chan YS, Dufresne A, Danquah MK. Review on nanoparticles and nanostructured materials: history, sources, toxicity and regulations. Beilstein J Nanotechnol. 2018;9:1050-1074. doi:10.3762/bjnano.9.98

3. Sorensen CM. Magnetism. In: Klabunde KJ, editor. Nanoscale Materials in Chemistry. New york: Wiley Interscience Publication; 2001:169-221.

4. Lu A-H, Salabas EL, Schüth F. Magnetic Nanoparticles: synthesis, Protection, Functionalization, and Application. Angewandte Chemie Int Edition. 2007;46(8):1222-1244. doi:10.1002/anie.200602866

5. Lee D-E, Koo H, Sun I-C, Ryu JH, Kim K, Kwon IC. Multifunctional nanoparticles for multimodal imaging and theragnosis. Chem Soc Rev. 2012;41(7):2656-2672. doi:10.1039/C2CS15261D

6. Hoang Thi TT, Nguyen Tran D-H, Bach LG, et al. Functional Magnetic Core-Shell System-Based Iron Oxide Nanoparticle Coated with Biocompatible Copolymer for Anticancer Drug Delivery. Pharmaceutics. 2019;11(3):120. doi:10.3390/pharmaceutics110301 20

7. Mukherjee A, Paul M, Mukherjee S. Recent Progress in the Theranostics Application of Nanomedicine in Lung Cancer. Cancers. 2019;11(5):597. doi:10.3390/cancers11050597

8. Odularu AT. Metal Nanoparticles: thermal Decomposition, Biomedicinal Applications to Cancer Treatment, and Future Perspectives. J Bioinorganic Chem App. 2018;2018:6. doi:10.1155/ 2018/9354708

9. Pelaz B, Alexiou C, Alvarez-Puebla RA, et al. Diverse Applications of Nanomedicine. ACS Nano. 2017;11(3):2313-2381. doi:10.1021/ acsnano.6b06040

10. Zhang E, Kircher MF, Koch M, Eliasson L, Goldberg SN, Renström E. Dynamic Magnetic Fields Remote-Control Apoptosis via Nanoparticle Rotation. ACS Nano. 2014;8(4):3192-3201. doi: $10.1021 / \mathrm{nn} 406302 \mathrm{j}$

11. Arriortua OK, Garaio E, Herrero de la Parte B, et al. Antitumor magnetic hyperthermia induced by RGD-functionalized $\mathrm{Fe}_{3} \mathrm{O}_{4}$ nanoparticles, in an experimental model of colorectal liver metastases. Beilstein $J$ Nanotechnol. 2016;7:1532-1542. doi:10.3762/ bjnano.7.147

12. Shao K, Singha S, Clemente-Casares $\mathrm{X}$, Tsai S, Yang $\mathrm{Y}$, Santamaria P. Nanoparticle-Based Immunotherapy for Cancer. ACS Nano. 2015;9(1):16-30. doi:10.1021/nn5062029

13. Mendes M, Sousa JJ, Pais A, Vitorino C. Targeted Theranostic Nanoparticles for Brain Tumor Treatment. Pharmaceutics. 2018;10 (4):181. doi:10.3390/pharmaceutics10040181

14. Mazaheri M, Eslahi N, Ordikhani F, Tamjid E, Simchi A. Nanomedicine applications in orthopedic medicine: state of the art Int J Nanomedicine. 2015;10:6039-6053. doi:10.2147/ijn.S73737

15. Silva CO, Pinho JO, Lopes JM, Almeida AJ, Gaspar MM, Reis C. Current Trends in Cancer Nanotheranostics: metallic, Polymeric, and Lipid-Based Systems. Pharmaceutics. 2019;11(1):22. doi:10.3390/ pharmaceutics 11010022

16. Kelkar SS, Reineke TM. Theranostics: combining Imaging and Therapy. Bioconjug Chem. 2011;22(10):1879-1903. doi:10.1021/ bc200151q

17. Wang LS, Chuang MC, Ho JA. Nanotheranostics-a review of recent publications. Int J Nanomedicine. 2012;7:4679-4695. doi:10.2147/ ijn.S33065

18. Depciuch J, Stec M, Maximenko A, et al. Synthesis method-dependent photothermal effects of colloidal solutions of platinum nanoparticles used in photothermal anticancer therapy. Applied Organometallic Chem. 2020;34(3):e5401. doi:10.1002/aoc.5401
19. Depciuch J, Stec M, Maximenko A, Pawlyta M, Baran J, ParlinskaWojtan M. Control of Arms of Au Stars Size and its Dependent Cytotoxicity and Photosensitizer Effects in Photothermal Anticancer Therapy. Int. J. Mol. Sci. 2019;20(20):5011. doi:10.3390/ijms20205011

20. Depciuch J, Stec M, Maximenko A, Baran J, Parlinska-Wojtan M. Temperature-controlled synthesis of hollow, porous gold nanoparticles with wide range light absorption. J Mater Sci. 2020;55 (12):5257-5267. doi:10.1007/s10853-020-04345-8

21. Depciuch J, Stec M, Kandler M, Baran J, Parlinska-Wojtan M. From spherical to bone-shaped gold nanoparticles-Time factor in the formation of $\mathrm{Au} \mathrm{NPs,} \mathrm{their} \mathrm{optical} \mathrm{and} \mathrm{photothermal} \mathrm{properties.}$ Photodiagnosis Photodyn Ther. 2020;30:101670. doi:10.1016/j. pdpdt.2020.101670

22. Wang Q, Cheng H, Peng H, Zhou H, Li PY, Langer R. Non-genetic engineering of cells for drug delivery and cell-based therapy. $A d v$ Drug Deliv Rev. 2015;91:125-140. doi:10.1016/j.addr.2014.12.003

23. Mousa SA, Bharali DJ. Nanotechnology-Based Detection and Targeted Therapy in Cancer: nano-Bio Paradigms and Applications. Cancers. 2011;3(3):2888-2903. doi:10.3390/cancers3032888

24. McBain SC, Yiu HHP, Dobson J. Magnetic nanoparticles for gene and drug delivery. Int $J$ Nanomedicine. 2008;3(2):169-180. doi:10.2147/ijn.s1608

25. Tietze R, Zaloga J, Unterweger H, et al. Magnetic nanoparticle-based drug delivery for cancer therapy. Biochem Biophys Res Commun. 2015;468(3):463-470. doi:10.1016/j.bbrc.2015.08.022

26. Messerschmidt C, Hofmann D, Kroeger A, Landfester K, Mailander V, Lieberwirth I. On the pathway of cellular uptake: new insight into the interaction between the cell membrane and very small nanoparticles. Beilstein $J$ Nanotechnol. 2016;7:1296-1311. doi:10.3762/bjnano.7.121

27. Salehi H, Derely L, Vegh A-G, et al. Label-free detection of anticancer drug paclitaxel in living cells by confocal Raman microscopy. Appl Phys Lett. 2013;102(11):113701. doi:10.1063/1.4794871

28. Salehi H, Calas-Bennasar I, Durand J-C, et al. Confocal Raman spectroscopy to monitor intracellular penetration of $\mathrm{TiO}_{2}$ nanoparticles. J Raman Spectroscopy. 2014;45(9):807-813. doi:10.1002/jrs.4561

29. Salehi H, Al-Arag S, Middendorp E, et al. Dental pulp stem cells used to deliver the anticancer drug paclitaxel. Stem Cell Res Ther. 2018;9(1):103. doi:10.1186/s13287-018-0831-3

30. Ansari SM, Bhor RD, Pai KR, et al. Cobalt nanoparticles for biomedical applications: facile synthesis, physiochemical characterization, cytotoxicity behavior and biocompatibility. Appl Surf Sci. 2017;414:171-187. doi:10.1016/j.apsusc.2017.03.002

31. Sarkar S, Mondal A, Giri N, Ray R. Spin glass like transition and the exchange bias effect in $\mathrm{Co}_{3} \mathrm{O}_{4}$ nanoparticles anchored onto graphene sheets. Phys Chem Chem Phys. 2019;21(1):260-267. doi:10.1039/ C8CP06659K

32. Salehi H, Al-Arag S, Middendorp E, Gergley C, Cuisinier F Stem cells as anticancer drug carrier to reduce the chemotherapy side effect. Proc. SPIE Imaging, Manipulation, and Analysis of Biomolecules, Cells, and Tissues XV. 2017;10068:1006805. doi:10.1117/12.2251994

33. Iglesia E. Design, synthesis, and use of cobalt-based Fischer-Tropsch synthesis catalysts. Appl Catal a Gen. 1997;161(1):59-78. doi:10.1016/S0926-860X(97)00186-5

34. Ghosh T, Dash SK, Chakraborty P, et al. Preparation of antiferromagnetic $\mathrm{Co}_{3} \mathrm{O}_{4}$ nanoparticles from two different precursors by pyrolytic method: in vitro antimicrobial activity. RSC $A d v$. 2014;4 (29):15022-15029. doi:10.1039/C3RA47769J

35. Abudayyak M, Gurkaynak TA, Özhan G. In vitro evaluation of cobalt oxide nanoparticle-induced toxicity. Angewandte Chemie Int Edition. 2017;33(8):646-654. doi:10.1177/0748233717706633

36. Alymov MI, Rubtsov NM, Seplyarskii BS, Kochetkov RA, Zelensky VA, Ankudinov AB. Combustion and passivation of nickel nanoparticles. Mendeleev Communications. 2017;27(6):631-633. doi:10.1016/j.mencom.2017.11.032 
37. Margeat O, Amiens C, Chaudret B, Lecante P, Benfield RE. Chemical Control of Structural and Magnetic Properties of Cobalt Nanoparticles. Chem Mater. 2005;17(1):107-111. doi:10.1021/ $\mathrm{cm} 048756 \mathrm{a}$

38. Jun Y-W, Seo J-W, Cheon J. Nanoscaling laws of magnetic nanoparticles and their applicabilities in biomedical sciences. Acc Chem Res. 2008;41(2):179-189. doi:10.1021/ar700121f

39. Liu S, Zhu J, Mastai Y, Felner I, Gedanken A. Preparation and characteristics of carbon nanotubes filled with cobalt. Chem Mater. 2000;12(8):2205-2211. doi:10.1021/cm000062o

40. Jaumann T, Ibrahim EMM, Hampel S, Maier D, Leonhardt A, Büchner B. The synthesis of superparamagnetic cobalt nanoparticles encapsulated in carbon through high-pressure CVD. Chem Vapor Deposition. 2013;19:(7-9):228-234. doi:10.1002/cvde.201207020

41. Cullity BD, Graham CD. Introduction to Magnetic Materials. 2nd. Wiley-IEEE Press; 2008. doi:10.1002/9780470386323

42. Hadjiev VG, Iliev MN, Vergilov IV. The Raman spectra of Co3O4. J Phys C: Solid State Phys. 1988;21(7):L199-L201. doi:10.1088/ 0022-3719/21/7/007

43. Jogade SM, Joshi PS, Jamadar BN, Sutrave DS. MOCVD of cobalt oxide using co-acetylacetonate as precursor: thin film deposition and study of physical properties. J Nano- Electron Phys. 2011;3 (1):203-211.

44. Pol SV, Pol VG, Seisenbaeva G, Kessler VG, Gedanken A. Stabilization of metastable face-centered cubic cobalt and the tetragonal phase of zirconia by a carbon shell: reaction under autogenic pressure at elevated temperature of $\mathrm{CoZr}_{2}(\mathrm{acac})_{2}(\mathrm{OiPr})_{8}$. Chem Mater. 2004;16(9):1793-1798. doi:10.1021/cm049830s

45. Tynan MK, Johnson DW, Dobson BP, Coleman KS. Formation of 3D graphene foams on soft templated metal monoliths. Nanoscale. 2016;8(27):13303-13310. doi:10.1039/C6NR02455F

46. Tvermoes BE, Unice KM, Paustenbach DJ, Finley BL, Otani JM, Galbraith DA. Effects and blood concentrations of cobalt after ingestion of $1 \mathrm{mg} / \mathrm{d}$ by human volunteers for $90 \mathrm{~d}$. Am J Clin Nutr. 2014;99(3):632-646. doi:10.3945/ajcn.113.071449
47. Sooklert K, Chattong S, Manotham K, et al. Cytoprotective effect of glutaraldehyde erythropoietin on HEK293 kidney cells after silver nanoparticle exposure. Int $J$ Nanomedicine. 2016;11:597-605. doi:10.2147/IJN.S95654

48. Chaves NL, Estrela-Lopis I, Böttner J, et al. Exploring cellular uptake of iron oxide nanoparticles associated with rhodium citrate in breast cancer cells. Int J Nanomedicine. 2017;12:5511-5523. doi:10.2147/ IJN.S141582

49. Kunwar A, Barik A, Mishra B, Rathinasamy K, Pandey R, Priyadarsini KI. Quantitative cellular uptake, localization and cytotoxicity of curcumin in normal and tumor cells. Biochimica et Biophysica Acta. 2008;1780(4):673-679. doi:10.1016/j.bbagen.2007. 11.016

50. Gal N, Massalha S, Samuelly-Nafta O, Weihs D. Effects of particle uptake, encapsulation, and localization in cancer cells on intracellular applications. Med Eng Phys. 2015;37(5):478-483. doi:10.1016/j. medengphy.2015.03.003

51. Parizi KB, Akin D, Wong HSP. Internalization of subcellular-scale microfabricated chips by healthy and cancer cells. PLoS One. 2018;13(3):e0194712. doi:10.1371/journal.pone.0194712

52. Surapaneni SK, Bashir S, Tikoo K. Gold nanoparticles-induced cytotoxicity in triple negative breast cancer involves different epigenetic alterations depending upon the surface charge. Sci Rep. 2018;8 (1):12295. doi:10.1038/s41598-018-30541-3

53. Layek B, Sadhukha T, Panyam J, Prabha S. Nano-Engineered Mesenchymal Stem Cells Increase Therapeutic Efficacy of Anticancer Drug Through True Active Tumor Targeting. Mol Cancer Ther. 2018;17(6):1196-1206. doi:10.1158/1535-7163.MCT17-0682

54. Yao S, Li X, Liu J, Sun Y, Wang Z, Jiang Y. Maximized nanodrug-loaded mesenchymal stem cells by a dual drug-loaded mode for the systemic treatment of metastatic lung cancer. Drug Deliv. 2017;24(1):1372-1383. doi:10.1080/10717544.2017.1375580
International Journal of Nanomedicine

\section{Publish your work in this journal}

The International Journal of Nanomedicine is an international, peerreviewed journal focusing on the application of nanotechnology in diagnostics, therapeutics, and drug delivery systems throughout the biomedical field. This journal is indexed on PubMed Central, MedLine, CAS, SciSearch ${ }^{\mathbb{}}$, Current Contents ${ }^{\mathbb{R}} /$ Clinical Medicine,
Journal Citation Reports/Science Edition, EMBase, Scopus and the Elsevier Bibliographic databases. The manuscript management system is completely online and includes a very quick and fair peer-review system, which is all easy to use. Visit http://www.dovepress.com/ testimonials.php to read real quotes from published authors. 